\title{
Influence of factors in the oral mucosa maturation pattern: a cross-sectional study applying multivariate analyses
}

\author{
Cristina da Silva Baumgart', Natália Batista Daroit', Bruna Jalfim Maraschin'1, Alex Haas², \\ Fernanda Visioli', Pantelis Varvaki Rados ${ }^{1}$
}

\author{
'Universidade Federal do Rio Grande do Sul - UFRS, School of Dentistry, Area of Oral Pathology, Porto Alegre, RS, Brazil \\ 2Universidade Federal do Rio Grande do Sul - UFRS, School of Dentistry, Area of Periodontology, Porto Alegre, RS, Brazil
}

\begin{abstract}
Aim: To evaluate the association between oral health status, socio-demographic and behavioral factors with the pattern of maturity of normal epithelial oral mucosa. Methods: Exfoliative cytology specimens were collected from 117 men from the border of the tongue and floor of the mouth on opposite sides. Cells were stained with the Papanicolaou method and classified into: anucleated, superficial cells with nuclei, intermediate and parabasal cells. Quantification was made by selecting the first 100 cells in each glass slide. Sociodemographic and behavioral variables were collected from a structured questionnaire. Oral health was analyzed by clinical examination, recording decayed, missing and filled teeth index (DMFT) and use of prostheses. Multivariable linear regression models were applied. Results: No significant differences for all studied variables influenced the pattern of maturation of the oral mucosa except for alcohol consumption. There was an increase of cell surface layers of the epithelium with the chronic use of alcohol. Conclusions: It is appropriate to use Papanicolaou cytopathological technique to analyze the maturation pattern of exposed subjects, with a strong recommendation for those who use alcohol - a risk factor for oral cancer, in which a change in the proportion of cell types is easily detected.
\end{abstract}

Keywords: Papanicolaou Test. Mouth Mucosa. Oral Health. Multivariate Analysis.

\section{Introduction}

Cytopathology is a diagnostic method that involves removing superficial mucosal cells by exfoliation for subsequent microscopic analysis ${ }^{1}$. With the introduction of quantitative techniques, oral cytology has become an important preventive exam for monitoring the oral mucosa exposed to carcinogens ${ }^{2-6}$. From these studies, it became apparent the individual nature of this type of evaluation, suggesting that some individuals are more susceptible than others to oral cancer development.

The cell type quantification of exfoliated oral mucosa by Papanicoloau staining is

Received for publication: May 05, 2016 Accepted: May 17, 2016

Correspondence to: Pantelis Varvaki Rados

Universidade Federal do Rio Grande do Sul Faculdade de Odontologia, Area de Patologia Oral. Rua Ramiro Barcelos, 2492/503 - CEP 90035-003

Porto Alegre, Rio Grande do Sul, Brazil

E-mail: pantelis@ufrgs.br one of the mentioned quantitative methods associated with cytopathology; this procedure allows the assessment of epithelial maturation process. Over time, many studies have sought to evaluate the influence of extrinsic factors (tobacco and alcohol consumption) and intrinsic factors such as sex and age in the process of oral epithelium maturation ${ }^{2,7}$. However, it remains uncertain whether the consumption of alcohol and tobacco, in addition to individual parameters modify the normal process of epithelial maturation of the oral cavity. Other factors that may change the pattern of oral epithelial maturation are oral hygiene status and socioeconomic factors, since they have been suggested as risk factors for the development of oral cancer $^{8-9}$. 
Thus, the aim of this study was to evaluate the influence of the oral health status, sociodemographic and behavioral factors on the maturation pattern of normal oral mucosa applying multivariate models.

\section{Material and methods}

\section{Study design and sample}

This was a cross-sectional observational study that evaluated male patients aged 25 years or older undergoing treatment at the School of Dentistry of the Federal University of Rio Grande do Sul, Brazil, who were considered eligible for the study. Visible lesions in the oral mucosa (except for periodontal disease), previous or current histories of malignant or benign tumors, radiotherapy and/ or chemotherapy, and use of fixed orthodontic appliances were the exclusion criteria.

The sample size was estimated from data obtained in a previous study ${ }^{2}$. The final sample comprised 117 men. Means and standard deviations of the number of superficial cells with nuclei in smokers/drinkers and non-smokers/non-drinkers were considered for sample calculation $(23.17 \pm 13.78$ and $18.00 \pm 11.91$, respectively). By estimation, 98 patients should be included to conduct the study with $80 \%$ power and alpha of $5 \%$.

\section{Ethical considerations}

The present study was conducted in accordance to the ethical guidelines set forth in the Declaration of Helsinki. The local Ethics Committee approved the study protocol and all patients signed an informed consent form prior to their inclusion in the study.

\section{Interview and Clinical examination}

Participants were interviewed using a structured questionnaire to gather demographic data, information about tobacco and alcohol habits, oral hygiene conditions, educational status, socio-economic variables and history of oral and systemic diseases.

A single researcher performed the oral examination, with the participant sitting in the proper position, artificial light, buccal mirror and explorer. The first physical examination was performed to detect visible oral lesions for exclusion. In their absence, the DMFT was recorded index according to the WHO criteria. Next, the prosthetic conditions were assessed. Dentures and removable appliances were removed for inspection. The number or lost teeth in each individual was derived from the DMFT index.

\section{Sample collection and cytopathological analysis}

Exfoliative cytology smears were collected from the border of tongue and floor of the mouth on opposite sides with four uniform brush turns by Cytobrush Plus ${ }^{\circledR}$. Smears were applied on a glass slide and then fixed in $99.6 \%$ alcohol. Samples were stained by the modified Papanicolaou technique ${ }^{2}$. One blinded observer examined the maturation pattern in all fields of each slide. One hundred wellformed and isolated cells were counted on each slide horizontally, from left to right, at 400x magnification. Cells were classified as anucleated, superficial with nuclei, intermediate and parabasal (Figure 1).

Intra-examiner reproducibility for classification of cell types was measured by the kappa statistic, considered acceptable when kappa is above 0.7 . Prior to the study, intra-examiner reproducibility was tested for quantification of 20 slides. During the study $10 \%$ of the sample was reassessed for reproducibility analysis.

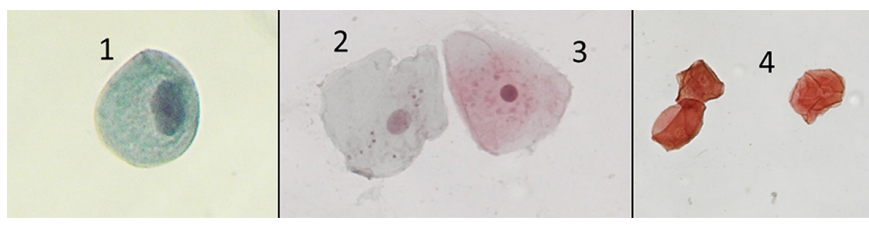

Fig.1. Cellular types of normal oral mucosa epithelium: 1) parabasal; 2) intermediate; 3 superficial with nuclei and 4) anucleated. (Papanicolaou method, 400x).

\section{Statistical Methods}

Independent Variables: Socio-demographic, behavioral and oral variables were evaluated in the present study. Age was categorized as $<50,50-59$ and $\geq 60$ years old. Skin color was dichotomized into white and non-white individuals. Marital status was categorized as married, single/divorced and widowed.

The total number of packs of cigarettes consumed in a lifetime was calculated by multiplying the number of cigarettes smoked per day by the number of years of habit, divided by 20 (1 pack). Subjects were classified into three groups: non-smokers ( 0 packyears), level 1 exposure ( $\leq 20$ pack-years) and level 2 exposure (>20 pack-years).

Daily alcohol consumption was calculated by multiplying the number of drinks consumed weekly by the average alcohol content of a glass of beer, wine or cachaça (a typical Brazilian drink distilled from sugarcane), divided by 7 days. The alcohol contents were estimated as $8 \mathrm{~g}$ in a glass of beer, $9.6 \mathrm{~g}$ in a glass of wine, and $8 \mathrm{~g}$ in a drink of cachaça. Drinkers were categorized into three groups: level 1 ( $\leq 3 \mathrm{~g} /$ day), level 2 ( $>3 \mathrm{~g}$ /day) and non-drinker.

Oral health conditions of the subjects were analyzed by DMFT index (decay, missing, filled teeth) and were classified in three categories according to the number of lost teeth: level 1 (1-19 teeth lost), level 2 ( $\geq 20$ teeth lost) and no tooth loss. For caries experience, individuals were grouped in two categories: Level 1 $(\mathrm{DMFT}=0)$ and level $2(\mathrm{DMFT} \geq 1)$.

Socio-economic status was assessed using a standard Brazilian economy classification (CCEB), which quantifies the home consumer goods. The subjects were grouped as low status or medium/high status and the cut off was 12 points. Educational level was categorized according to the number of education years, as follows: low level ( $\leq 4$ years), intermediate level (5-10 years of education), and high level ( $\geq 11$ years of education).

Statistical analyses: Cell types were expressed in mean percentage and standard error separately for the border of the tongue and floor of the mouth. Comparisons between means according to the independent variables (socio-demographic, behavioral and oral) were performed using Wald tests, adjusting for multiple comparisons if required.

Predictive models of multivariable linear regression were 
applied by entering all independent variables of interest in the model. Interactions and multicollinearity were evaluated and were not found. Data analysis was conducted using Stata software (StataCorp., version 10 for Macintosh). The individual was the analytical unit. The level of significance was set at $5 \%$.

\section{Results}

The characteristics of the participants of the study are presented in Table 1. The study sample comprised mostly Caucasian men under 50 years of age, married, from low socioeconomic status and medium educational level. Regarding oral status, the majority of individuals reported brushing teeth at least once a day and did not use mouthwashes. Almost half the patients said they had never smoked. Among smokers or former smokers, the percentage of heavy smokers was high. Regarding alcohol consumption, almost $40 \%$ of the patients never ingested alcohol beverages and equal amount use more than $3 \mathrm{~g}$ of alcohol per day (Table 1).

Tables 2 and 3 present the means and standard errors of exfoliated squamous cells percentage from the border of the tongue and the floor of the mouth, respectively, according to the independent variables. On the border of tongue, $75 \%$ of the cells were intermediate, $20 \%$ were superficial cells with nuclei, $5 \%$ were anucleated cells. Parabasal cells were seldom observed. A significantly higher percentage of superficial and anucleated cells was observed in drinker subjects compared to non-drinker individuals. For intermediate cells, the percentage was significantly lower in drinkers compared to those who did not drink alcoholic beverages (Table 2).

Table 3 presents the pattern of maturation of the floor of the mouth, where approximately $82.5 \%$ of the cells were intermediate, $15 \%$ were superficial cells with nuclei, $2.5 \%$ were anucleated, whereas parabasal cells were rarely observed. Evaluating the studied variables, again only alcohol consumption was statistically significant, with differences between drinkers and non-drinkers. Similar to the tongue, intermediate cells were found in smaller proportions and anucleated cells in larger number in individuals who drank alcohol often.

Tables 4 and 5 show the multivariate linear regression models for the association between the percentage of cell types on the border of tongue and floor of mouth, respectively, as well as the independent variables. Significant associations were observed for consumption of alcoholic beverages, which showed increase of anucleated cells and superficial cells with nuclei, as well as decrease of intermediate cells with increased alcohol consumption.

\section{Discussion}

Some risk factors contributing to oral cancer, such as smoking and alcohol, are already well established in the literature ${ }^{10-11}$. Analysis of individuals exposed to these factors using Papanicolaou test showed alterations in maturation pattern of cells ${ }^{2,12}$. This study aimed to evaluate other possible variables that could influence the pattern of maturation of oral epithelial cells, such as oral health and sociodemographic factors. To our knowledge, this study is the first to assess by cytopathology, the influence of oral health, behavioral and sociodemographic factors on maturation pattern of oral mucosa using multilevel modeling.

Table 1 - Absolute distribution and percentage of sociodemographic and behavioral status of the participants.

\begin{tabular}{|c|c|c|}
\hline Variables & $n$ & $\%$ \\
\hline \multicolumn{3}{|l|}{ Age } \\
\hline$<50$ years & 61 & 52.1 \\
\hline $50-59$ years & 30 & 25.7 \\
\hline$\geq 60$ years & 26 & 22.2 \\
\hline \multicolumn{3}{|l|}{ Skin color } \\
\hline White & 81 & 69.2 \\
\hline Non-white & 36 & 30.8 \\
\hline \multicolumn{3}{|l|}{ Marital status } \\
\hline Married & 67 & 57.3 \\
\hline Single/divorced & 48 & 41.0 \\
\hline Widowed & 2 & 1.7 \\
\hline \multicolumn{3}{|l|}{ Socio-economic status } \\
\hline Medium/High & 53 & 45.3 \\
\hline Low & 64 & 54.7 \\
\hline \multicolumn{3}{|l|}{ Educational level } \\
\hline High ( $\geq 11$ education years) & 44 & 37.6 \\
\hline Intermediate (5-10 education years) & 50 & 42.7 \\
\hline Low ( $\leq 4$ education years) & 23 & 19.7 \\
\hline \multicolumn{3}{|l|}{ Brush teeth } \\
\hline$<1$ time/day & 3 & 2.6 \\
\hline$\geq 1$ time/day & 114 & 97.4 \\
\hline \multicolumn{3}{|l|}{ Use mouthwashes } \\
\hline Yes & 25 & 21.4 \\
\hline No & 92 & 78.6 \\
\hline \multicolumn{3}{|l|}{ Visited the dentist } \\
\hline$<1$ year & 57 & 48.7 \\
\hline$\geq 1$ year & 60 & 51.3 \\
\hline \multicolumn{3}{|l|}{ Smoke } \\
\hline Current smoker & 25 & 21.4 \\
\hline Former smoker & 30 & 25.6 \\
\hline Never smoked & 62 & 53.0 \\
\hline \multicolumn{3}{|l|}{ Exposed smoke } \\
\hline Never smoked & 62 & 53.0 \\
\hline Smoked $\leq 20$ pack-years & 34 & 29.1 \\
\hline Smoked > 20 pack-years & 21 & 17.9 \\
\hline \multicolumn{3}{|l|}{ Alcohol consumption } \\
\hline Never ingested alcohol & 46 & 39.3 \\
\hline Drink $\leq 3 g$ & 24 & 20.5 \\
\hline Drink $>3 g$ & 47 & 40.2 \\
\hline Total & 117 & 100.0 \\
\hline
\end{tabular}

In order to assist the early detection of this altered tissue even before clinical lesion, when a biopsy contraindicated, oral exfoliative cytopathology can be used, performing tests such as Papanicolaou stain (PAP). Oral cytopathology is a fast, easy to perform, non-invasive and inexpensive technique; which allows to evaluate changes in cytological pattern maturation of normal oral mucosa, inferring risk groups and assisting in the prevention of these factors ${ }^{1}$. 
Table 2 - Mean distribution (standard error) the percentage of the type of cells on the border of tongue according to independent variables.

\begin{tabular}{|c|c|c|c|c|c|c|c|c|}
\hline Variables & Anucleated & $p^{*}$ & Supnuclei** & $p^{*}$ & Intermediate & $p^{*}$ & Parabasal & $p^{*}$ \\
\hline \multicolumn{9}{|l|}{ Age } \\
\hline$<50$ years & $4.97 \pm 0.33$ & Ref. & $20.37 \pm 0.38$ & Ref. & $74.84 \pm 0.53$ & Ref. & $0.00 \pm 0.00$ & Ref. \\
\hline $50-59$ years & $5.37 \pm 0.51$ & 0.51 & $20.10 \pm 0.52$ & 0.67 & $74.53 \pm 0.81$ & 0.76 & $0.00 \pm 0.00$ & - \\
\hline $60+$ years & $5.57 \pm 0.53$ & 0.33 & $19.77 \pm 0.66$ & 0.43 & $74.58 \pm 0.84$ & 0.80 & $0.08 \pm 0.05$ & 0.15 \\
\hline \multicolumn{9}{|l|}{ Skin color } \\
\hline White & $5.35 \pm 0.31$ & Ref. & $20.37 \pm 0.34$ & Ref. & $74.40 \pm 0.50$ & Ref. & $0.01 \pm 0.01$ & Ref. \\
\hline Non-white & $4.89 \pm 0.38$ & 0.35 & $19.72 \pm 0.48$ & 0.27 & $75.36 \pm 0.59$ & 0.22 & $0.03 \pm 0.03$ & 0.61 \\
\hline \multicolumn{9}{|l|}{ Socio-economic status } \\
\hline High & $5.15 \pm 0.38$ & Ref. & $20.34 \pm 0.40$ & Ref. & $74.51 \pm 0.58$ & Ref. & $0.02 \pm 0.02$ & Ref. \\
\hline Low & $5.25 \pm 0.32$ & 0.84 & $20.03 \pm 0.39$ & 0.58 & $74.86 \pm 0.53$ & 0.66 & $0.02 \pm 0.02$ & 0.89 \\
\hline \multicolumn{9}{|l|}{ Educational level } \\
\hline High & $5.39 \pm 0.38$ & Ref. & $20.36 \pm 0.41$ & Ref. & $74.48 \pm 0.58$ & Ref. & $0.00 \pm 0.00$ & Ref. \\
\hline Intermediate & $5.04 \pm 0.38$ & 0.52 & $20.28 \pm 0.46$ & 0.89 & $74.66 \pm 0.62$ & 0.83 & $0.04 \pm 0.03$ & 0.16 \\
\hline Low & $5.22 \pm 0.56$ & 0.80 & $19.56 \pm 0.64$ & 0.30 & $75.22 \pm 0.94$ & 0.51 & $0.00 \pm 0.00$ & - \\
\hline \multicolumn{9}{|l|}{ Alcohol consumption } \\
\hline Never ingested alcohol & $4.43 \pm 0.36$ & Ref. & $19.35 \pm 0.45$ & Ref. & $76.22 \pm 0.58$ & Ref. & $0.02 \pm 0.02$ & Ref. \\
\hline Drink $\leq 3 \mathrm{~g}$ & $6.04 \pm 0.56$ & 0.02 & $20.42 \pm 0.68$ & 0.19 & $73.96 \pm 0.85$ & 0.03 & $0.00 \pm 0.00$ & 0.32 \\
\hline Drink $>3 g$ & $5.53 \pm 0.38$ & 0.04 & $20.85 \pm 0.39$ & 0.01 & $73.60 \pm 0.60$ & 0.01 & $0.02 \pm 0.02$ & 0.99 \\
\hline \multicolumn{9}{|l|}{ Exposed Smoke } \\
\hline Never smoked & $5.00 \pm 0.33$ & Ref. & $20.24 \pm 0.40$ & Ref. & $74.74 \pm 0.52$ & Ref. & $0.02 \pm 0.02$ & Ref. \\
\hline Smoked $\leq 20$ pack-years & $5.47 \pm 0.42$ & 0.38 & $20.21 \pm 0.49$ & 0.95 & $74.35 \pm 0.72$ & 0.66 & $0.00 \pm 0.00$ & 0.32 \\
\hline Smoked $>20$ pack-years & $5.38 \pm 0.67$ & 0.61 & $19.90 \pm 0.65$ & 0.66 & $75.14 \pm 1.04$ & 0.73 & $0.05 \pm 0.05$ & 0.53 \\
\hline \multicolumn{9}{|l|}{ Smoke + Alcohol } \\
\hline Never smoked/never drink & $4.82 \pm 0.49$ & Ref. & $19.71 \pm 0.58$ & Ref. & $75.43 \pm 0.74$ & Ref. & $0.04 \pm 0.04$ & Ref. \\
\hline Smoke or Drink intermediate & $5.29 \pm 0.48$ & 0.50 & $19.75 \pm 0.65$ & 0.97 & $75.00 \pm 0.81$ & 0.70 & $0.00 \pm 0.00$ & 0.32 \\
\hline Smoke or drink hard & $5.34 \pm 0.35$ & 0.39 & $20.57 \pm 0.35$ & 0.21 & $74.23 \pm 0.55$ & 0.20 & $0.02 \pm 0.02$ & 0.62 \\
\hline \multicolumn{9}{|l|}{ Use mouthwashes } \\
\hline No & $5.17 \pm 0.27$ & Ref. & $20.17 \pm 0.31$ & Ref. & $74.74 \pm 0.43$ & Ref. & $0.02 \pm 0.02$ & Ref. \\
\hline Yes & $5.32 \pm 0.56$ & 0.82 & $20.16 \pm 0.67$ & 0.98 & $74.56 \pm 0.94$ & 0.86 & $0.00 \pm 0.00$ & 0.16 \\
\hline \multicolumn{9}{|l|}{ Teeth lost } \\
\hline 0 tooth & $4.08 \pm 0.45$ & Ref. & $20.00 \pm 0.47$ & Ref. & $75.19 \pm 0.70$ & Ref. & $0.00 \pm 0.00$ & Ref. \\
\hline $1-19$ teeth & $5.38 \pm 0.30$ & 0.30 & $20.31 \pm 0.37$ & 0.61 & $74.46 \pm 0.50$ & 0.39 & $0.01 \pm 0.01$ & 0.32 \\
\hline $20+$ teeth & $5.21 \pm 0.87$ & 0.68 & $19.86 \pm 0.88$ & 0.89 & $74.86 \pm 1.25$ & 0.81 & $0.07 \pm 0.07$ & 0.32 \\
\hline \multicolumn{9}{|l|}{ Caries experience } \\
\hline $\mathrm{DMFT} \leq 8$ & $4.88 \pm 0.38$ & Ref. & $20.32 \pm 0.39$ & Ref. & $74.98 \pm 0.64$ & Ref. & $0.02 \pm 0.02$ & Ref. \\
\hline $\mathrm{DMFT} \geq 9$ & $5.46 \pm 0.31$ & 0.24 & $20.04 \pm 0.38$ & 0.61 & $74.47 \pm 0.47$ & 0.53 & $0.02 \pm 0.02$ & 0.87 \\
\hline \multicolumn{9}{|l|}{ Use of oral prosthesis } \\
\hline Not use & $5.17 \pm 0.28$ & Ref. & $20.43 \pm 0.32$ & Ref. & $74.40 \pm 0.46$ & Ref. & $0.01 \pm 0.01$ & Ref. \\
\hline Partial removable prosthesis & $5.47 \pm 0.56$ & 0.63 & $19.63 \pm 0.63$ & 0.26 & $75.89 \pm 0.79$ & 0.59 & $0.00 \pm 0.00$ & 0.31 \\
\hline Total prosthesis & $5.07 \pm 0.86$ & 0.92 & $19.36 \pm 0.95$ & 0.29 & $76.21 \pm 1.38$ & 0.22 & $0.07 \pm 0.07$ & 0.42 \\
\hline Total & $5.21 \pm 0.24$ & & $20.17 \pm 0.28$ & & $74.70 \pm 0.39$ & & $0.02 \pm 0.01$ & \\
\hline
\end{tabular}

${ }^{*}$ Comparisons of the reference group (Ref.).

${ }^{* *}$ superficial cells with nuclei.

In these studies, the only statistically significant variable associated with alterations in maturation pattern was the use of alcohol, which resulted in an increase of surface cell layers of the epithelium (anucleated and superficial with nuclei) in individuals exposed to alcohol. Our results contradict the previous literature. One study evaluated the cell maturation pattern of individuals exposed to tobacco and alcohol observed a smaller number of superficial cells with nuclei compared to the non-exposed group ${ }^{2}$. We consider that these results differ from our study for some reasons: first, because alcohol consumption was not evaluated alone, but synergistically with tobacco; further, in our study was performed a multivariate analysis, thus, it can control other variables that could be biases. Another reason is that in our study a greater number of participants was assessed compared with the above-mentioned study. 
Table 3 - Mean distribution (standard error) the percentage of the type of cells on the floor of the mouth according to independent variables.

\begin{tabular}{|c|c|c|c|c|c|c|c|c|}
\hline Variables & Anucleated & $\mathrm{p}^{*}$ & Supnuclei** & $p^{*}$ & Intermediate & $\mathrm{p}^{*}$ & Parabasal & $\mathrm{p}^{*}$ \\
\hline \multicolumn{9}{|l|}{ Age } \\
\hline$<50$ years & $2.31 \pm 0.30$ & Ref. & $15.46 \pm 0.53$ & Ref. & $82.06 \pm 0.65$ & Ref. & $0.00 \pm 0.00$ & Ref. \\
\hline $50-59$ years & $2.17 \pm 0.35$ & 0.75 & $14.33 \pm 0.81$ & 0.25 & $83.53 \pm 0.97$ & 0.21 & $0.03 \pm 0.03$ & 0.31 \\
\hline $60+$ years & $2.40 \pm 0.39$ & 0.82 & $15.88 \pm 0.87$ & 0.68 & $81.65 \pm 0.97$ & 0.72 & $0.04 \pm 0.04$ & 0.32 \\
\hline \multicolumn{9}{|l|}{ Skin color } \\
\hline White & $2.21 \pm 0.24$ & Ref. & $15.17 \pm 0.48$ & Ref. & $82.48 \pm 0.56$ & Ref. & $0.03 \pm 0.02$ & Ref. \\
\hline Non-white & $2.50 \pm 0.37$ & 0.51 & $15.47 \pm 0.71$ & 0.72 & $82.06 \pm 0.89$ & 0.69 & $0.00 \pm 0.00$ & 0.16 \\
\hline \multicolumn{9}{|l|}{ Socio-economic status } \\
\hline High & $2.06 \pm 0.25$ & Ref. & $15.64 \pm 0.55$ & Ref. & $82.30 \pm 0.59$ & Ref. & $0.04 \pm 0.03$ & Ref. \\
\hline Low & $2.50 \pm 0.30$ & 0.26 & $14.95 \pm 0.56$ & 0.38 & $82.39 \pm 0.71$ & 0.93 & $0.00 \pm 0.00$ & 0.16 \\
\hline \multicolumn{9}{|l|}{ Educational level } \\
\hline High & $2.43 \pm 0.33$ & Ref. & $15.41 \pm 0.68$ & Ref. & $82.16 \pm 0.73$ & Ref. & $0.02 \pm 0.02$ & Ref. \\
\hline Intermediate & $2.34 \pm 0.33$ & 0.84 & $15.14 \pm 0.55$ & 0.76 & $82.34 \pm 0.74$ & 0.86 & $0.02 \pm 0.02$ & 0.93 \\
\hline Low & $1.96 \pm 0.35$ & 0.32 & $15.26 \pm 1.01$ & 0.90 & $82.73 \pm 1.14$ & 0.67 & $0.00 \pm 0.00$ & 0.32 \\
\hline \multicolumn{9}{|l|}{ Alcohol consumption } \\
\hline Never ingested alcohol & $1.63 \pm 0.25$ & Ref. & $14.74 \pm 0.68$ & Ref. & $83.61 \pm 0.75$ & Ref. & $0.02 \pm 0.02$ & Ref. \\
\hline Drink $\leq 3 g$ & $2.75 \pm 0.49$ & 0.04 & $15.08 \pm 0.72$ & 0.73 & $81.79 \pm 1.04$ & 0.16 & $0.04 \pm 0.04$ & 0.67 \\
\hline Drink $>3 g$ & $2.72 \pm 0.33$ & 0.01 & $15.87 \pm 0.63$ & 0.22 & $81.40 \pm 0.72$ & 0.04 & $0.00 \pm 0.00$ & 0.32 \\
\hline \multicolumn{9}{|l|}{ Exposed Smoke } \\
\hline Never smoked & $2.18 \pm 0.28$ & Ref. & $15.18 \pm 0.54$ & Ref. & $82.65 \pm 0.65$ & Ref. & $0.02 \pm 0.02$ & Ref \\
\hline Smoked $\leq 20$ pack-years & $2.47 \pm 0.38$ & 0.53 & $16.47 \pm 0.70$ & 0.15 & $80.76 \pm 0.80$ & 0.07 & $0.00 \pm 0.00$ & 0.32 \\
\hline Smoked $>20$ pack-years & $2.38 \pm 0.47$ & 0.71 & $13.57 \pm 0.90$ & 0.13 & $84.05 \pm 1.13$ & 0.29 & $0.05 \pm 0.05$ & 0.53 \\
\hline \multicolumn{9}{|l|}{ Smoke + Alcohol } \\
\hline Never smoked/never drink & $1.82 \pm 0.35$ & Ref. & $15.46 \pm 0.86$ & Ref. & $82.64 \pm 0.98$ & Ref. & $0.04 \pm 0.04$ & Ref. \\
\hline Smoke or Drink intermediate & $2.29 \pm 0.42$ & 0.40 & $15.14 \pm 0.70$ & 0.77 & $82.28 \pm 0.93$ & 0.79 & $0.00 \pm 0.00$ & 0.32 \\
\hline Smoke or drink hard & $2.52 \pm 0.28$ & 0.12 & $15.23 \pm 0.57$ & 0.82 & $82.25 \pm 0.67$ & 0.74 & $0.02 \pm 0.02$ & 0.62 \\
\hline \multicolumn{9}{|l|}{ Use mouthwashes } \\
\hline No & $2.39 \pm 0.23$ & Ref. & $15.04 \pm 0.42$ & Ref. & $82.43 \pm 0.53$ & Ref. & $0.02 \pm 0.02$ & Ref. \\
\hline Yes & $1.96 \pm 0.34$ & 0.31 & $16.08 \pm 1.04$ & 0.36 & $82.04 \pm 1.05$ & 0.74 & $0.00 \pm 0.00$ & 0.16 \\
\hline \multicolumn{9}{|l|}{ Teeth lost } \\
\hline 0 tooth & $1.81 \pm 0.32$ & Ref. & $15.45 \pm 0.65$ & Ref. & $82.74 \pm 0.73$ & Ref. & $0.00 \pm 0.00$ & Ref. \\
\hline 1-19 teeth & $2.44 \pm 0.27$ & 0.13 & $15.29 \pm 0.55$ & 0.85 & $82.11 \pm 0.66$ & 0.52 & $0.03 \pm 0.02$ & 0.16 \\
\hline $20+$ teeth & $2.64 \pm 0.58$ & 0.21 & $14.71 \pm 1.00$ & 0.54 & $82.71 \pm 1.31$ & 0.99 & $0.00 \pm 0.00$ & - \\
\hline \multicolumn{9}{|l|}{ Caries experience } \\
\hline $\mathrm{DMFT} \leq 8$ & $2.32 \pm 0.27$ & Ref. & $15.15 \pm 0.60$ & Ref. & $82.51 \pm 0.68$ & Ref. & $0 \pm 0$ & Ref. \\
\hline DMFT $\geq 9$ & $2.27 \pm 0.28$ & 0.89 & $15.35 \pm 0.60$ & 0.80 & $82.21 \pm 0.65$ & 0.75 & $-0.30 \pm-0.21$ & 0.15 \\
\hline \multicolumn{9}{|l|}{ Use of oral prosthesis } \\
\hline Not use & $2.35 \pm 0.25$ & Ref. & $15.51 \pm 0.48$ & Ref. & $82.12 \pm 0.56$ & Ref. & $0.02 \pm 0.02$ & Ref. \\
\hline Partial removable prosthesis & $1.68 \pm 0.36$ & 0.13 & $15.26 \pm 1.04$ & 0.83 & $82.53 \pm 1.26$ & 0.76 & $0.00 \pm 0.00$ & 0.16 \\
\hline Total prosthesis & $2.79 \pm 0.53$ & 0.47 & $13.79 \pm 0.90$ & 0.09 & $83.5 \pm 1.26$ & 0.32 & $0.00 \pm 0.00$ & 0.16 \\
\hline Total & $2.30 \pm 0.20$ & & $15.26 \pm 0.40$ & & $82.35 \pm 0.47$ & & $0.02 \pm 0.01$ & \\
\hline
\end{tabular}

Another study evaluated the proportion of cell types of the oral mucosa of individuals who used daily Listerine ${ }^{\circledR}$ mouthwash with $26.9 \%$ of alcohol for 6 months compared to the group that used the mouthwash without alcohol. Only outer cells of the epithelium (superficial and intermediate) were found in both groups, with no statistical differences between the pattern of epithelium maturation using a mouthwash with or without alcohol ${ }^{13}$. However, that study ${ }^{13}$ does not detail the criteria used for cell type analysis, nor how many cells were analyzed. Another factor that explains the difference observed in our study was that individuals evaluated in our study were chronic alcohol users for years and several times a day. 
Table 4 - Multivariate linear regression model of the association between the percentage of cells at the border of tongue and the independent variables.

\begin{tabular}{|c|c|c|c|c|c|c|c|c|}
\hline Variables & Anucleated & & Supnuclei* & & Intermediate & & Parabasal & \\
\hline & $\beta \pm S E^{* *}$ & $p$ & $\beta \pm S E^{* *}$ & $p$ & $\beta \pm S E^{\star *}$ & $p$ & $\beta \pm S E^{* *}$ & $p$ \\
\hline Age & $0.01 \pm 0.02$ & 0.85 & $0.01 \pm 0.02$ & 0.67 & $-0.27 \pm 0.03$ & 0.44 & $0.01 \pm 0.01$ & 0.16 \\
\hline \multicolumn{9}{|l|}{ Skin color } \\
\hline White & Ref. & & Ref. & & Ref. & & Ref. & \\
\hline Non-white & $-0.25 \pm 0.56$ & 0.66 & $-0.57 \pm 0.65$ & 0.37 & $0.61 \pm 0.88$ & 0.49 & $0.01 \pm 0.02$ & 0.61 \\
\hline \multicolumn{9}{|l|}{ Exposed Smoke } \\
\hline Never smoked & Ref. & & Ref. & & Ref. & & Ref. & \\
\hline Smoked $\leq 20$ pack-years & $0.05 \pm 0.58$ & 0.92 & $-0.33 \pm 0.68$ & 0.62 & $0.27 \pm 0.92$ & 0.76 & $-0.01 \pm 0.02$ & 0.68 \\
\hline Smoked $>20$ pack-years & $0.14 \pm 0.75$ & 0.84 & $-0.16 \pm 0.88$ & 0.85 & $0.29 \pm 1.19$ & 0.80 & & \\
\hline \multicolumn{9}{|l|}{ Alcohol consumption } \\
\hline Never ingested alcohol & Ref. & & Ref. & & Ref. & & Ref. & \\
\hline Drink $\leq 3 g$ & $1.69 \pm 0.67$ & 0.01 & $1.03 \pm 0.78$ & 0.19 & $-2.31 \pm 1.07$ & 0.03 & $-0.01 \pm 0.03$ & 0.66 \\
\hline Drink $>3 g$ & $1.40 \pm 0.58$ & 0.01 & $1.37 \pm 0.67$ & 0.04 & $-2.92 \pm 0.92$ & 0.00 & $0.01 \pm 0.02$ & 0.69 \\
\hline \multicolumn{9}{|l|}{ Use mouthwashes } \\
\hline No & Ref. & & Ref. & & Ref. & & Ref. & \\
\hline Yes & $0.24 \pm 0.61$ & 0.69 & $-0.04 \pm 0.70$ & 0.95 & $-0.19 \pm 0.96$ & 0.84 & $-0.01 \pm 0.03$ & 0.59 \\
\hline DMFT index & $0.06 \pm 0.04$ & 0.09 & $-0.02 \pm 0.04$ & 0.53 & $-0.05 \pm 0.06$ & 0.38 & $-0.01 \pm 0.01$ & 0.84 \\
\hline \multicolumn{9}{|l|}{ Use of oral prosthesis } \\
\hline Not use & Ref. & & Ref. & & Ref. & & Ref. & \\
\hline Partial removable prosthesis & $-0.03 \pm 0.78$ & 0.96 & $-0.46 \pm 0.91$ & 0.61 & $0.82 \pm 1.24$ & 0.50 & $-0.03 \pm 0.03$ & 0.33 \\
\hline Total prosthesis & $-0.33 \pm 0.89$ & 0.71 & $-0.85 \pm 1.04$ & 0.41 & $2.05 \pm 1.42$ & 0.15 & $0.02 \pm 0.04$ & 0.63 \\
\hline $\mathrm{R}^{2}$ & 0.02 & & -0.01 & & 0.03 & & -0.03 & \\
\hline
\end{tabular}

Table 5 - Multivariate linear regression model of the association between the percentage of cells on the floor of the mouth and the independent variables.

\begin{tabular}{|c|c|c|c|c|c|c|c|c|}
\hline Variables & Anucleated & & Supnuclei ${ }^{*}$ & & Intermediate & & Parabasal & \\
\hline & $\beta \pm S E^{* *}$ & $\mathrm{p}$ & $\beta \pm S E^{* *}$ & $p$ & $\beta \pm S E^{* *}$ & $p$ & $\beta \pm S E^{* *}$ & $p$ \\
\hline Age & $0.02 \pm 0.01$ & 0.21 & $0.06 \pm 0.03$ & 0.07 & $-0.08 \pm 0.04$ & 0.05 & $0.01 \pm 0.01$ & 0.25 \\
\hline \multicolumn{9}{|l|}{ Skin color } \\
\hline White & Ref. & & Ref. & & Ref. & & Ref. & \\
\hline Non-white & $0.41 \pm 0.45$ & 0.36 & $0.48 \pm 0.91$ & 0.59 & $-0.63 \pm 1.08$ & 0.55 & $-0.01 \pm 0.02$ & 0.53 \\
\hline \multicolumn{9}{|l|}{ Exposed Smoke } \\
\hline Never smoked & Ref. & & Ref. & & Ref. & & Ref. & \\
\hline Smoked $\leq 20$ pack-years & $0.21 \pm 0.47$ & 0.64 & $1.07 \pm 0.95$ & 0.26 & $-1.49 \pm 1.12$ & 0.18 & $-0.01 \pm 0.02$ & 0.51 \\
\hline Smoked $>20$ pack-years & $0.02 \pm 0.61$ & 0.97 & $-1.68 \pm 1.23$ & 0.17 & $1.77 \pm 1.45$ & 0.22 & $0.03 \pm 0.03$ & 0.35 \\
\hline \multicolumn{9}{|l|}{ Alcohol consumption } \\
\hline Never ingested alcohol & Ref. & & Ref. & & Ref. & & Ref. & \\
\hline Drink $\leq 3 g$ & $1.10 \pm 0.54$ & 0.04 & $0.26 \pm 1.10$ & 0.80 & $-1.70 \pm 1.30$ & 0.19 & $0.02 \pm 0.03$ & 0.51 \\
\hline Drink $>3 g$ & $1.16 \pm 0.47$ & 0.01 & $1.24 \pm 0.94$ & 0.19 & $-2.29 \pm 1.12$ & 0.04 & $-0.01 \pm 0.02$ & 0.92 \\
\hline \multicolumn{9}{|l|}{ Use mouthwashes } \\
\hline No & Ref. & & Ref. & & Ref. & & Ref. & \\
\hline Yes & $-0.32 \pm 0.49$ & 0.51 & $0.69 \pm 0.99$ & 0.48 & $-0.05 \pm 1.17$ & 0.96 & $-0.02 \pm 0.03$ & 0.47 \\
\hline DMFT index & $-0.01 \pm 0.03$ & 0.68 & $-0.02 \pm 0.06$ & 0.72 & $0.04 \pm 0.07$ & 0.60 & $0.01 \pm 0.01$ & 0.12 \\
\hline \multicolumn{9}{|l|}{ Use of oral prosthesis } \\
\hline Not use & Ref. & & Ref. & & Ref & & Ref & \\
\hline Partial removable prosthesis & $-0.89 \pm 0.63$ & 0.16 & $-1.06 \pm 1.28$ & 0.41 & $1.12 \pm 1.51$ & 0.42 & $-0.06 \pm 0.03$ & 0.47 \\
\hline Total prosthesis & $0.20 \pm 0.72$ & 0.77 & $-1.63 \pm 1.46$ & 0.26 & $1.30 \pm 1.73$ & 0.45 & $-0.08 \pm 0.04$ & 0.06 \\
\hline$\overline{R^{2}}$ & 0.02 & & 0.01 & & 0.03 & & 0.01 & \\
\hline
\end{tabular}


Some researches using cytopathology observed the influence of the chronic use of ethanol on the cells of oral mucosa. Reis et al. ${ }^{14}$ (2006) showed a significant increase in micronucleus, abnormal nucleus/cytoplasm ratio, pyknosis, karyorrhexis and karyolysis in exposed mucosa. Webber et al. ${ }^{6}$ (2016) evaluated by Feulgen staining nuclear changes in cells from the border of the tongue and the floor of the mouth of alcoholics. The last cited site showed a higher frequency of karyorrhexis and this suggests higher degrees of keratinization. This statement agrees with our results, taking into account the alcohol variable, we found more cells of the superficial layers, which suggests an organism reaction as protection against aggression, maybe with increased keratinization.

In contradiction, smoking, the greatest risk factor for oral cancer alone did not significantly affect the maturation pattern of the oral mucosa. The results are conflicting in the literature on this subject. Burzlaff and $\mathrm{Gedoz}^{2-3}$ found an increased number of surface nucleated cells in patients exposed to tobacco smoke.

The sites chosen for the collection of smears were tongue and floor of the mouth because in Brazil these locations are the most frequently affected by oral squamous cell carcinoma ${ }^{15-16}$. In our results it is possible to see a clear distinction of cell types (anucleated, superficial cells with nuclei, intermediate and parabasal) at each site, approximately with $5 \%, 20 \%, 75 \%, 0 \%$ on the tongue, and $2.5 \%$, $15 \%, 82.5 \%, 0 \%$ on the floor of the mouth, respectively (Tables 2 and 3). It is important to know the particular pattern of each site under normal conditions the floor of mouth is less keratinized than the border of tongue, so it presents a lower amount of surface and more intermediate cells $\mathrm{s}^{6,17}$ - to detect possible changes in this cellular types proportion when exposed to a carcinogenic factor.

Some studies correlated low income with higher incidence of oral cancer ${ }^{18-19}$,for this reason sociodemographic factors were included as independent variables. Because cytopathology has been used as a monitoring tool of cytogenetic changes, we decided to include these factors in our study. Madani ${ }^{19}$ reported that individuals with low educational level have 3.3 more chances of developing the disease than those with higher schooling. Regarding the results of our study, the pattern of the oral epithelium maturation had no significant differences comparing the different ranges of economic and educational level.

The association between the status of oral health and head and neck cancer has been widely studied; these studies suggest that poor oral hygiene can contribute to an increased risk of this pathology ${ }^{20-21}$. In the present study there was no change in cell maturation pattern in relation to oral health status, which disagrees with the idea that oral microorganisms can increase inflammatory cytokines level and change the complex metabolic pathways, triggering the process of carcinogenesis ${ }^{22}$.

The use of Papanicolaou cytopathological method is appropriate to analyze the proportion of exfoliated cells as the maturation pattern of healthy male subjects. The maturation pattern of the buccal mucosa cells was not affected by the studied variables, except by the intake of alcoholic beverages.

\section{References}

1. Kazanowska K, Hałon A, Radwan-Oczko M. The role and application of exfoliative cytology in the diagnosis of oral mucosa pathology - contemporary knowledge with review of the literature. Adv Clin Exp Med 2014; 23: 299-305.

2. Burzlaff JB, Bohrer PL, Paiva RL, Visioli F, Sant'Ana Filho M, da Silva $\mathrm{VD}$, et al. Exposure to alcohol or tobacco affects the pattern of maturation in oral mucosal cells: a cytohistological study. Cytopathology. 2007; 18 : 367-75.

3. Gedoz L, Lauxen I, Sant'Ana Filho M, Rados PV. Proliferative activity in clinically healthy oral mucosa exposed to tobacco smoking and alcohol: a longitudinal study using the AgNOR staining technique. Anal Quant Cytol Histol. 2007; 29: 231-8.

4. Ahmed HG, Omer AS, Abd Algaffar SA. Cytological study of exfoliative buccal mucosal cells of Qat chewers in Yemen. Diagn Cytopathol. 2011; 39: 796-800.

5. Oliveira LU, Lima CF, Salgado MA, Balducci I, Almeida JD. Comparative study of oral mucosa micronuclei in smokers and alcoholic smokers. Ana Quant Cytol Histol. 2012; 34: 9-14.

6. Webber LP, Pellicioli A, Magnusson AS, Danilevicz CK, Bueno CC, Sant'Ana Filho M, et al. Nuclear changes in oral mucosa of alcoholics and crack cocaine users. Hum Exp Toxicol. 2016; 35: 184-93.

7. Cowpe JG, Longmore RB, Green MW. Quantitative exfoliative cytology of normal oral squames: an age, site and sex-related survey. J R Soc Med. 1985; 78: 995-1004.

8. Cowpe JG, Longmore RB, Green MW. Quantitative exfoliative cytology of normal oral squames: an age, site and sex-related survey. J R Soc Med. 1985; 78: 995-1004.

9. Ahrens W, Pohlabeln H, Foraita R, Nelis M, Lagiou P, Lagiou A, et al. Oral health, dental care and mouthwash associated with upper aerodigestive tract cancer risk in Europe: the ARCAGE study. Oral Oncol. 2014; 50 : 616-25.

10. Andrade JO, Santos CA, Oliveira MC. Associated factors with oral cancer: a study of case control in a population of the Brazil's Northeast. Rev Bras Epidemiol. 2015; 18: 894-905.

11. Sanner T, Grimsrud TK. Nicotine: Carcinogenicity and Effects on Response to Cancer Treatment - A Review. Front Oncol. 2015; 5: 196.

12. Abdelaziz MS, Osman TE. Detection of Cytomorphological Changes in Oral Mucosa among Alcoholics and Cigarette Smokers. Oman Med J. 2011; 26: 349-52.

13. Bagan JV, Vera-Sempere F, Marzal C, Carcelén AP, Martí-Bonmati E, Bagan L. Cytological changes in the oral mucosa after use of a mouth rinse with alcohol: A prospective double blind control study. Med Oral Patol Oral Cir Bucal. 2012; 17: e956-e961.

14. Reis SR, do Espírito Santo AR, Andrade MG, Sadigursky M. Cytologic alterations in the oral mucosa after chronic exposure to ethanol. Braz Oral Res. 2006; 20: 97-102.

15. Gervásio OL, Dutra RA, Tartaglia SM, Vasconcellos WA, Barbosa AA, Aguiar MC. Oral squamous cell carcinoma: A retrospective study of 740 cases in a Brazilian Population. Braz Dent J. 2001; 12: 57-61.

16. Durazzo MD, de Araujo CE, Brandão Neto JS, Potenza AS, Costa P, Takeda F, et al. Clinical and epidemiological features of oral cancer in a medical school teaching hospital from 1994 to 2002: increasing incidence in women, predominance of advanced local disease, and low incidence of neck metastases. Clinics. 2005; 60: 293-8.

17. Braga FL, Meneguzzi RD, Paiva RL, Rados PV. [Cytopathology evaluation of the oral mucosa of smokers and nonsmokers]. Odonto Cien. 2004; 19 : 157-63. [Portuguese]

18. Boing AF, Antunes JL. [Socioeconomic conditions and head and neck cancer: a systematic literature review]. Cien Saude Colet. 2011; 16 : 
615-22. [Portuguese].

19. Madani AH, Dikshit M, Bhaduri D, Jahromi AS, Aghamolaei T. Relationship between selected socio-demographic factors and cancer of oral cavity - a case control study. Cancer Inform. 2010; 9: 163-8.

20. Meyer MS, Joshipura K, Giovannucci E, Michaud DS. A review of the relationship between tooth loss, periodontal disease, and cancer. Cancer Causes Control. 2008; 19: 895-907.

21. Coussens LM, Werb Z. Inflammation and cancer. Nature 2002; 420:860-7.

22. Rajeev R, Choudhary K, Panda S, Gandhi N. Role of bacteria in oral carcinogenesis. South Asian J Cancer. 2012; 1: 78-83. 